\title{
Intracranial aneurysm growth: consistency of morphological changes
}

\author{
Eva L. Leemans, MSc, ${ }^{1,2}$ Bart M. W. Cornelissen, MSc, ${ }^{1-3}$ Miran Said, MD, ${ }^{1}$ \\ René van den Berg, MD, PhD, ${ }^{2}$ Cornelis H. Slump, PhD, ${ }^{3}$ Henk A. Marquering, PhD, ${ }^{1,2}$ and \\ Charles B. L. M. Majoie, MD, PhD²
}

Departments of ${ }^{1}$ Biomedical Engineering and Physics, and ${ }^{2}$ Radiology and Nuclear Medicine, Amsterdam UMC, University of Amsterdam, Amsterdam; and ${ }^{3}$ Department of Robotics and Mechatronics, University of Twente, Enschede, The Netherlands

\begin{abstract}
OBJECTIVE Previous studies have shown a relation between growth and rupture of intracranial aneurysms. Additionally, several morphological characteristics are frequently measured to estimate rupture risk. Little is known about how the rupture risk is associated with morphological characteristic changes during growth. The aim of this study was to provide insights into how morphological characteristics, associated with rupture, change during an aneurysm's growth.

METHODS The authors retrospectively identified patients with longitudinal MRA images of unruptured growing aneurysms. The MRA images had an in-plane resolution of $0.2-0.5 \mathrm{~mm}$ and a slice thickness of $0.2-0.75 \mathrm{~mm}$. Therefore, growth was defined as an increase of at least $0.5 \mathrm{~mm}$ in two directions or $1 \mathrm{~mm}$ in one direction. Using the MRA images, the authors semiautomatically segmented the aneurysm and the perianeurysmal vasculature. Twelve morphological characteristics were automatically measured. These characteristics were related to size (diameter, height, width, neck diameter, volume, surface area, aspect ratio, height-width ratio, and bottleneck factor) and shape (ellipticity index, nonsphericity index, and undulation index) of the aneurysm. Morphological characteristics before and after growth were compared using the Wilcoxon signed-rank test.

RESULTS The authors included 31 patients with 38 growing aneurysms. The aneurysms' growth was detected after a mean of 218 weeks (range 23-567 weeks). A significant increase was seen in all size-related characteristics, and the bottleneck factor also significantly increased (from a median of 1.00 [IQR 0.85-1.04] to 1.03 [IQR 0.93-1.18]), while the ellipticity index decreased (from a median of 0.26 [IQR $0.25-0.28$ ] to 0.25 [IQR $0.24-0.26]$ ). The changes in size ratios and shape indices varied largely among patients. Larger aneurysms more often showed an increase in shape ratios.

CONCLUSIONS Although aneurysm growth, size-related characteristics, bottleneck factor, and ellipticity index changed significantly during growth, most size ratios and shape indices showed inconsistent changes among aneurysms. This suggests that, for an accurate rupture prediction, morphological parameters need to be reassessed after growth.

https://thejns.org/doi/abs/10.3171/2019.4.FOCUS1987
\end{abstract}

KEYWORDS aneurysm; morphology; growth; MRA; rupture

A $\mathrm{N}$ increasing number of unruptured aneurysms are being detected due to improvements in and the wide availability of imaging..$^{15}$ Only a small number of intracranial aneurysms will eventually rupture. Rupture often leads to death or severe disability and poses a considerable clinical and socioeconomic burden. ${ }^{17}$ Because aneurysm treatment also poses considerable risks, ${ }^{22,25}$ the risk of rupture must outweigh treatment risk. Current guidelines recommend that the following factors be taken into account when deciding upon treatment: size, irregularity, location, aneurysm growth, family history of subarachnoid hemorrhage (SAH), age, hypertension, ${ }^{23,36}$ previous $\mathrm{SAH}$, and the presence of comorbidities. ${ }^{7,16,23,34,36}$

If an aneurysm is left untreated, periodic follow-up imaging of the aneurysm is recommended. It has been reported that approximately $10 \%$ of the aneurysms show growth on follow-up imaging. ${ }^{12}$ Aneurysm growth is associated with an increasing risk of rupture, as growing aneurysms have proven to be unstable..$^{36}$ Growth patterns differ-for instance, some grow globally, whereas oth-

ABBREVIATIONS AR = aspect ratio; BNF = bottleneck factor; EI = ellipticity index; HWR = height-width ratio; NSI = nonsphericity index; SAH = subarachnoid hemorrhage; $\mathrm{UI}=$ undulation index

ACCOMPANYING EDITORIAL DOI: 10.3171/2019.4.FOCUS19332.

SUBMITTED February 14, 2019. ACCEPTED April 11, 2019

INCLUDE WHEN CITING DOI: 10.3171/2019.4.FOCUS1987. 
ers form a bleb. Different growth patterns might have a different rupture risk as different pathological processes underlie the growth. ${ }^{28}$ Thus, a greater understanding of aneurysm growth is needed to improve the management of unruptured aneurysms.

Next to size, several other aneurysm morphological characteristics, such as aspect ratio (AR) and ellipticity index (EI), are frequently used to predict which aneurysms

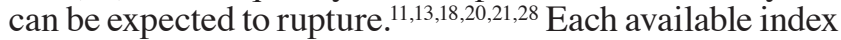
describes a different aspect of the aneurysm's morphology. These characteristics may change as the aneurysm grows. Not much is known about the dynamics of these characteristics during the time that the aneurysm is growing. Nonetheless, these characteristics are used to predict the risk of rupture at a given moment in time. The aim of this study was to provide insights into how morphological characteristics, associated with rupture, change during the aneurysm's growth.

\section{Methods}

\section{Patient Population}

We retrospectively included all patients with saccular unruptured intracranial aneurysms and longitudinal imaging performed from 2012 to 2016 at our tertiary care referral center. In this population of 253 patients with 333 aneurysms, 55 aneurysms were marked as growing aneurysms based on the imaging report by the neuroradiologist. These reports documented a change in size of more than $1 \mathrm{~mm}$ in one direction, $0.5 \mathrm{~mm}$ in two directions, or did not show a significant change in shape..$^{5}$ Longitudinal imaging was performed with either CTA or MRA. The MRA consisted of 3D time-of-flight or contrast-enhanced MRA. We excluded all patients with only CTA studies available $(\mathrm{n}=9)$, as most CTA images contained slices of $>0.75 \mathrm{~mm}$ and thus no accurate segmentation could be made. Additionally, we excluded 11 scans because of poor imaging quality $(n=2)$, different MRA sequences $(n=1)$, no available time-of-flight imaging $(\mathrm{n}=4)$, and imaging conducted after treatment $(\mathrm{n}=2)$. As a result, this study included 31 patients with 38 growing aneurysms. All included MRA images were made on a Philips Medical Systems scanner. The scan parameters were as follows: TR 20-47 msec, TE 1.5-6.4 msec, in-plane resolution 0.2-0.5 $\mathrm{mm}$, and slice thickness $0.2-0.75 \mathrm{~mm}$. The requirement for informed consent was waived by the institution's review board because no diagnostic tests other than routine clinical imaging were used in this study.

\section{Morphology}

To assess aneurysm morphology, we used the following longitudinal images: baseline images, all images showing growth, and final image of the untreated aneurysm. As a result, for each patient the aneurysm morphology was determined at 2 to 4 moments in time. We focused the analysis on the baseline imaging and the final available imaging. The other moments in time were used to evaluate when growth was first detected and whether an aneurysm continued to grow after initial detection.

We have segmented the aneurysm and perianeurysmal vasculature on the available images using the Vascular
Modeling Toolkit (www.vmtk.org). ${ }^{2}$ Both thresholding and a Sato vesselness filter were used for the automated segmentation. Each model was visually inspected by an expert with more than 2 years' experience in neurovascular imaging (E.L.L.). If needed, the segmentations were manually corrected to reduce inaccuracies due to imaging artifacts such as flow dispersion, saturation, and partial voluming. The manual corrections were performed with ITK-SNAP 3.6.0 (www.itksnap.org).

In 4 aneurysms (11 models), because it was difficult to differentiate the aneurysm from its surrounding vessels, these models were also corrected by an experienced neuroradiologist (C.B.L.M.M.). Subsequently, the surfaces of the resultant segmentations were smoothed with the builtin Taubin-smoothing algorithm of the Vascular Modeling Toolkit, thereby removing high-frequency artifacts without shrinking the surface..$^{33}$ The following smoothing parameters were used: passband factor of 0.05 and 20 iterations.

To calculate each aneurysm's morphological characteristics, the aneurysm volume and neck plane were automatically determined. First, the original parent vessel was reconstructed. We used the parent vessel reconstruction algorithm devised by Ford et al. ${ }^{14}$ This parent vessel reconstruction consists of creating multiple centerlines in the parent vessel, determining where the aneurysm deviates from the parent vessel, and reconstructing the original vessel using the Voronoi diagram (Fig. 1A-F). With the delineation of the parent artery, the aneurysm surface was extracted (Fig. 1). The aneurysm surface is here defined as the part of the total surface that does not coincide with the parent vessel surface.

In current clinical practice, several morphological characteristics are measured after defining the aneurysm neck plane. The neck plane is detected using the methodology reported by Piccinelli et al. ${ }^{27}$ By defining planes at multiple orientations on the centerline, the optimal neck plane can be found (Fig. 1G), which is the plane with the smallest area and which is the closest to, but not intersecting, the parent vessel.

\section{Morphological Characteristics}

With the delineation of the aneurysm surface and the neck plane, the morphological characteristics were automatically calculated. The characteristics can be divided into 4 groups: 2D size characteristics, size ratios, 3D size characteristics, and shape characteristics. In addition to the shape characteristics, the irregularity was also documented on the baseline imaging by an experienced neuroradiologist. All characteristics were measured in accordance with the definitions proposed by Raghavan et al. ${ }^{28}$

The 2D size characteristics consist of the maximum diameter, height, and width. These were measured as depicted in Fig. 1F. The maximum diameter is the maximum distance found between two points on the aneurysm surface. Aneurysm height is defined as the longest distance from the center of the neck plane to the aneurysm dome. Aneurysm width depicts the longest distance perpendicular to the measurement of the aneurysm height. In addition to the 2D size characteristics, two 3D size characteristics are determined: aneurysm volume and surface area. 

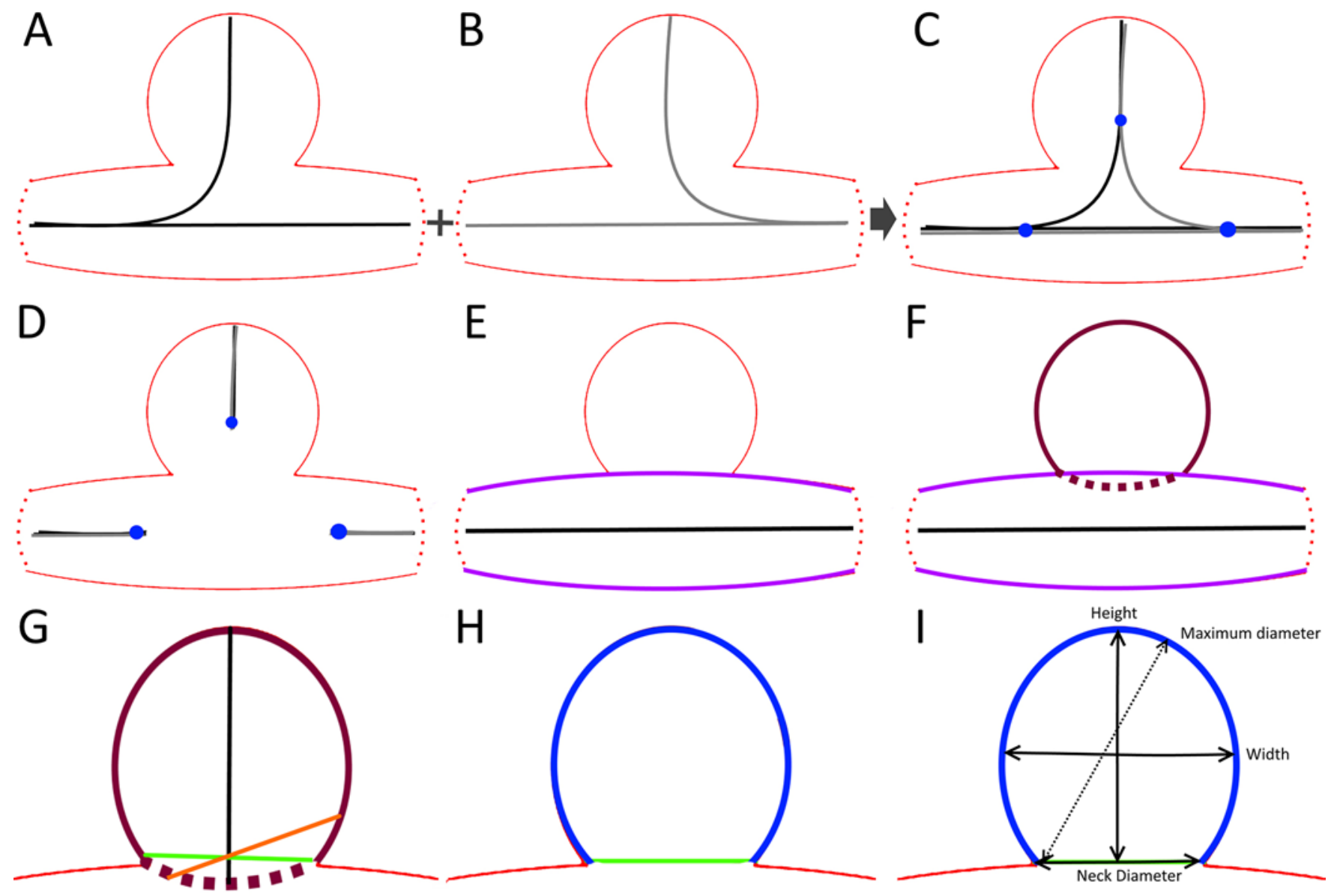

FIG. 1. Parent vessel reconstruction and aneurysm assessment. A: Forward centerline. B: Backward centerline. C: Combination of centerline and identification of the clipping points (b/ue). D: Clipping at the clipping points. E: Reconstruction of the parent vessel, and therefore this cannot be a neck plane; the green plane does not intersect and is marked as a possible neck plane. F: Extraction of the aneurysm surface (brown) from the parent vessel. G: Testing multiple possible neck-planes along the aneurysm centerline. H: Selection of the perfect neck plane (green) and extraction of the aneurysm surface from the neck onwards. I: Evaluating aneurysm morphology.

With these 2D size characteristics, several size ratios were determined: height-width ratio (HWR), bottleneck factor (BNF), and AR. The HWR depicts the difference between the height and the width of the aneurysm. The $\mathrm{BNF}$ is the ratio between the width and neck diameter; it therefore shows if an aneurysm has a small or a broad base. The AR is the ratio between the height and the neck diameter; it thus shows how elongated the aneurysm is.

The last characteristics are the shape characteristics: EI, nonsphericity index (NSI), and undulation index (UI). For these indices, the convex hull is determined. The convex hull of an aneurysm is the smallest convex volume that encloses the aneurysm volume. The EI characterizes the deviation of the aneurysm convex hull, as described by the convex hull volume $\left(V_{c h}\right)$ and surface area $\left(S A_{c h}\right)$, from a perfect sphere $\left[E I=18 \pi^{1 / 3}\left(V_{c h}^{2 / 3}\right) /\left(S A_{c h}\right)\right]$. The NSI is similar to the EI, but instead of the convex hull volume and surface area, the volume and surface area of the aneurysm are used. It is therefore influenced by both ellipticity and surface irregularities (undulations). The UI captures the aneurysm irregularity, as it compares the aneurysm vol- ume with the volume of the convex hull $\left[U I=1-V / V_{c h}\right]$; a completely regular aneurysm without any undulations will have an UI of 0 .

\section{Statistical Analysis}

All variables were tested for normality using a Shapiro-Wilk test. Normally distributed values are reported as mean $\pm \mathrm{SD}$, nonnormally distributed values are reported as median and interquartile range (IQR 25\%-75\%). Morphological characteristics at baseline were compared with the characteristics at the final imaging follow-up using a paired t-test for normally distributed variables and a Wilcoxon signed-rank test for nonnormally distributed variables. A $\mathrm{p}$ value $<0.05$ was considered statistically significant.

\section{Results}

We included 31 patients with 38 growing aneurysms with a total of 118 MRI studies. Two examples are displayed in Fig. 2. The clinical characteristics can be found 

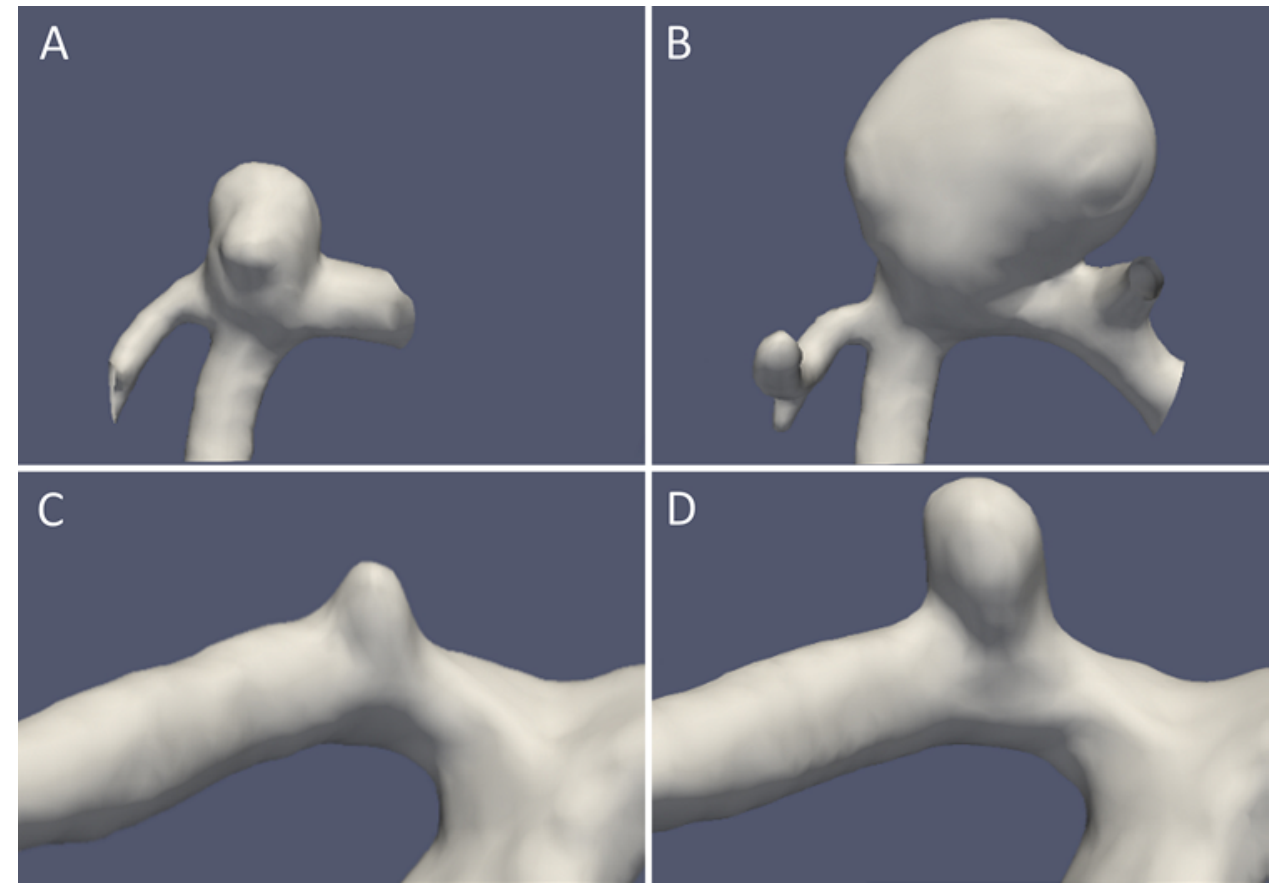

FIG. 2. Examples of aneurysms before ( $A$ and $C$ ) and after growth ( $B$ and $D)$. The aneurysm in $A$ and $B$ also shows a clear change in shape.

in Table 1. Most patients had multiple aneurysms $(n=21$, $68 \%)$ and were female $(n=27,87 \%)$. In about one-third of the cases, the patient had suffered a previous SAH $(n=9$, $29 \%$ ) of another aneurysm.

Aneurysm growth was detected after 218 weeks (range 23-567 weeks). Twelve of the growing aneurysms (32\%) were treated, and one (3\%) ruptured during follow-up. The aneurysms were treated between 12 and 283 weeks after the detection of growth (4 within 6 months from the detection of growth). The one that ruptured did so 28 weeks af-

TABLE 1. Clinical characteristics in 31 patients with 38 growing aneurysms

\begin{tabular}{lc}
\hline \multicolumn{1}{c}{ Characteristic } & Value $(\%)$ \\
\hline Sex (female) & $27(87 \%)$ \\
\hline Aneurysm multiplicity & $21(68 \%)$ \\
\hline Age at baseline (yrs) & $51 \pm 11$ \\
\hline Previous SAH & $9(29 \%)$ \\
\hline Location of the growing aneurysm & $8(21 \%)$ \\
\hline ICA & $8(21 \%)$ \\
\hline ACA/ACoA & $16(42 \%)$ \\
\hline MCA & $6(16 \%)$ \\
\hline PCoA/posterior circulation & $32(84 \%)$ \\
\hline Aneurysm shape at baseline (regular)
\end{tabular}

$\mathrm{ACA}=$ anterior cerebral artery; $\mathrm{ACo}=$ anterior communicating artery; $\mathrm{ICA}=$ internal carotid artery; $\mathrm{MCA}=$ middle cerebral artery; $\mathrm{PCOA}=$ posterior communicating artery.

Values are presented as the number (\%) of patients or aneurysms or as the mean \pm SD. ter the detection of growth. Blebs were formed in 2 aneurysms $(5 \%)$, while 8 aneurysms $(21 \%)$ had a clear change in shape (i.e., new multiple lobulations or wall protrusions). On average, the aneurysms grew $1.8 \pm 2.1 \mathrm{~mm}$, and no differences were seen among aneurysm locations. In 11 aneurysms (29\%), growth was detected on multiple scans after baseline imaging; however, for 13 lesions (34\%), we only had two sets of images, and thus in these cases we could not determine if these aneurysms were still unstable after growth.

\section{Morphological Characteristics}

Nine of the 12 morphological characteristics changed significantly after growth (Table 2). Most of these characteristics were associated with the aneurysm size. A significant increase was seen in diameter, width, height, neck diameter, BNF, volume, and surface area, whereas the EI and UI decreased. On average, the aneurysm width increased more than its height, but the change in HWR was insignificant. The increase in BNF indicates that, during growth, the aneurysm neck grew slower or less than the aneurysm width. The decrease in EI indicates that the aneurysm's convex hull deviates more from a perfect sphere and thus that the aneurysm becomes less spherical or has more undulations.

As can be seen in Fig. 3, the 2D and 3D size-related characteristics increase in most patients during the lesion's growth. However, the change in width is more consistent with the aneurysm's diameter than the change in its height. No clear trends were seen for the size ratios (AR, BNF, and HWR); in some aneurysms these ratios increased, but in other aneurysms these ratios decreased. In most aneurysms the EI decreased during growth. However, some an- 
TABLE 2. Morphological characteristics at baseline and final imaging

\begin{tabular}{llccc}
\hline \multicolumn{1}{c}{ Characteristic } & Baseline Imaging & Final Imaging & Difference & $p$ Value \\
\hline Diameter $(\mathrm{mm})$ & $5.0(3.4-7.0)$ & $6.9(4.9-9.0)$ & $1.8(2.1)$ & 0.01 \\
\hline Width $(\mathrm{mm})$ & $3.6(2.6-5.5)$ & $5.04(3.9-7.9)$ & $1.6(1.7)$ & 0.003 \\
\hline Height $(\mathrm{mm})$ & $2.2(1.5-3.9)$ & $3.3(2.1-5.2)$ & $1.0(1.6)$ & 0.02 \\
\hline Neck diameter $(\mathrm{mm})$ & $3.6(2.5-5.5)$ & $4.58(3.2-6.9)$ & $1.2(1.2)$ & 0.01 \\
\hline BNF & $1.00(0.85-1.04)$ & $1.03(0.93-1.18)$ & $0.09(0.35)$ & 0.05 \\
\hline AR & $0.63(0.50-0.77)$ & $0.68(0.57-0.87)$ & $0.06(0.20)$ & 0.06 \\
\hline HWR & $0.63(0.54-0.78)$ & $0.68(0.49-0.81)$ & $0.01(0.3)$ & 0.60 \\
\hline Volume $\left(\mathrm{mm}^{3}\right)$ & $13.9(5.0-51.3)$ & $44.0(13.4-105.7)$ & $41.7(66.8)$ & 0.01 \\
\hline Surface area $\left(\mathrm{mm}^{2}\right)$ & $31.9(16.8-74.7)$ & $71.8(29.5-120.5)$ & $36.4(46.6)$ & 0.01 \\
\hline EI & $0.26(0.25-0.28)$ & $0.25(0.24-0.26)$ & $-0.01(0.02)$ & 0.02 \\
\hline NSI & $0.19(0.17-0.22)$ & $0.19(0.17-0.22)$ & $0.00(0.04)$ & 0.82 \\
\hline UI & $0.23(0.14-0.35)$ & $0.18(0.13-0.22)$ & $-0.05(0.11)$ & 0.02 \\
\hline
\end{tabular}

Values are presented as the median (IQR) or the mean (SD).

eurysms exhibited a stable or increased EI. A similar trend was seen for the UI.

Figure 4 shows for each morphological characteristic the relation of the magnitude of the measured change with the baseline diameter. No dependencies on baseline diameter were seen for the 2D size characteristics and the size indices. The aneurysm volume and surface area showed larger changes, with an increasing baseline diameter. Additionally, the shape indices seemed to decrease with smaller baseline diameters but increase with larger diameters.

\section{Discussion}

This study evaluated to what extent commonly measured morphological characteristics change during aneurysm growth. A significant increase was seen in the $2 \mathrm{D}$ and 3D size-related characteristics and the BNF during growth of intracranial aneurysms. The EI and UI significantly decreased. Both the size ratios and shape indices showed inconsistent trends; in some aneurysms these ratios increased, but in other aneurysms the values decreased. Based on the Bland-Altman plots, the shape indices seemed to decrease when baseline diameters were smaller, but to increase when baseline diameters were larger.

The aneurysm growth rate in our patient population (19\%) is in line with other studies that reported growth rates between $4 \%$ and $45 \% .{ }^{4}$ Several studies showed that an aneurysm's size and irregularity are a strong predictor for growth. ${ }^{3}$ Our study showed that the change in most parameters was not dependent on the size of the lesion at baseline, but a larger aneurysm diameter at baseline often resulted in an increase instead of decrease in the shape indices (EI, NSI, and UI). What this indicates is that larger aneurysms are more likely to become irregularly shaped or to form a bleb during growth, whereas smaller aneurysms often become more regular and spherical. As aneurysm irregularity is related to both growth ${ }^{5}$ and rupture, ${ }^{19,21}$ the rupture risk of these smaller aneurysms might decrease during growth. It is yet unknown whether the other presented rupture risk-related characteristics predict growth. Nevertheless, our study provides insights into what happens to these features during growth.

Previous studies reported higher $\mathrm{AR},,^{11,13,28,38} \mathrm{BNF},{ }^{13}$ HWR,,$^{13}$ UI, ${ }^{11,28,37}$ and NSI ${ }^{11,28,37}$ and a lower EI ${ }^{11,28,37}$ in ruptured aneurysms. Growing aneurysms have proven to be unstable and more prone to rupture. ${ }^{36}$ Therefore, one might expect that after growth aneurysms have a morphology similar to the ruptured aneurysms of previous studies. Our study only showed a significant increase for the BNF and a significantly lower EI. Thus, most of these rupturerelated characteristics did not change significantly after growth. This might be because many studies use the images obtained after rupture to determine the morphology, but it has been shown that the morphology changes after rupture. ${ }^{31}$ Additionally, we did observe inconsistent changes in morphology among the various aneurysms. The difference in morphological change among these aneurysms might indicate a different rupture risk. Some aneurysms stabilize after growth. Although aneurysm growth is related to their rupture, not every growing aneurysm ruptures. Further research is needed to evaluate whether the difference in growth patterns can be used to predict rupture. With morphological changes, the hemodynamic environment also changes, which could further influence the rupture risk. ${ }^{8,9,32,35}$ Because aneurysm hemodynamics could influence the aneurysm wall, ${ }^{24}$ these changes might (de)stabilize the aneurysm.

Currently, the rupture risk is often estimated based on a single moment in time. Such an approach neglects the dynamic aspect of an aneurysm. Therefore, evaluating rupture risk based on a single moment in time might be unreliable. As our study shows, there is a wide variability in the changes in morphology during growth. Therefore these indices might not predict rupture at a single point in time. Reassessing the parameters during and after growth likely gives more extensive information on the aneurysm stability and rupture risk.

Only a small portion of the aneurysms in our data set 

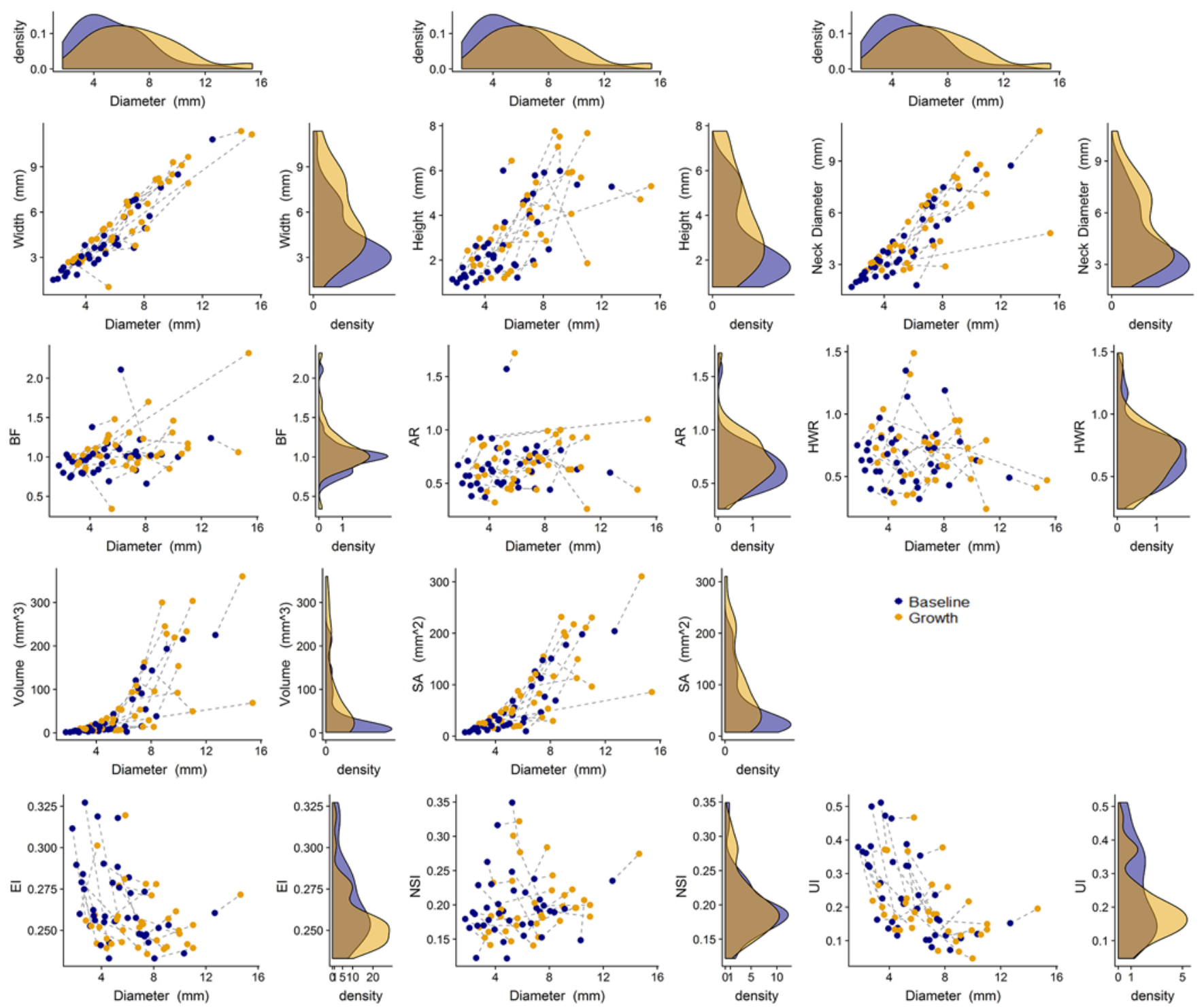

FIG. 3. Scatter and density plots for each morphological characteristic versus diameter. The density plots of the diameter are displayed on the first row. The density plots of each characteristic are displayed vertically (columns 2, 4, and 6). Blue dots and density plots represent the baseline values, orange dots and density plots represent the values after growth (at the time of the final imaging). The dotted lines connect the baseline and final value of one aneurysm. BF = bottleneck factor; $\mathrm{SA}=$ surface area.

were treated after growth due to the estimated low risk of rupture after growth. A large portion of the aneurysms were at the middle cerebral artery $(42 \%)$, and the diameter after growth was often below $7 \mathrm{~mm}$. Although some patients had suffered an earlier SAH from another aneurysm, the 5-year cumulative rupture risk is still small $(0.4 \%-0.9 \%$ [as estimated based on the PHASES score $]^{16}$ ). Since the risk posed by endovascular treatment in these patients was higher (mortality rate $1 \%-2 \%$ and morbidity rate $4 \%-10 \%{ }^{25,29}$ ), most patients were not treated and were followed with additional imaging. However, one should be aware that, during growth, the neck diameter often also increases, and therefore an aneurysm with increased size does not automatically imply that endovascular procedures will be easier. ${ }^{10}$ When choosing conser- vative treatment after growth of the aneurysm has been documented, shorter intervals between imaging can be recommended.

A limitation of this study is that the measurements of the morphological characteristics depend on the segmentations that are performed. ${ }^{6}$ Due to slow-flow artifacts the aneurysm lumen might appear smaller or incomplete on TOF MRA. To optimize accuracy, all segmentations were performed on MRA and were subsequently inspected by an experienced neuroradiologist. Additionally, the same imaging sequence was used at baseline and follow-up imaging; thus, the increase in size was independent of the imaging method. More accurate measurements can be obtained with 3D rotational angiography due to the high resolution and signal-to-noise ratios. ${ }^{1}$ However, follow-up 

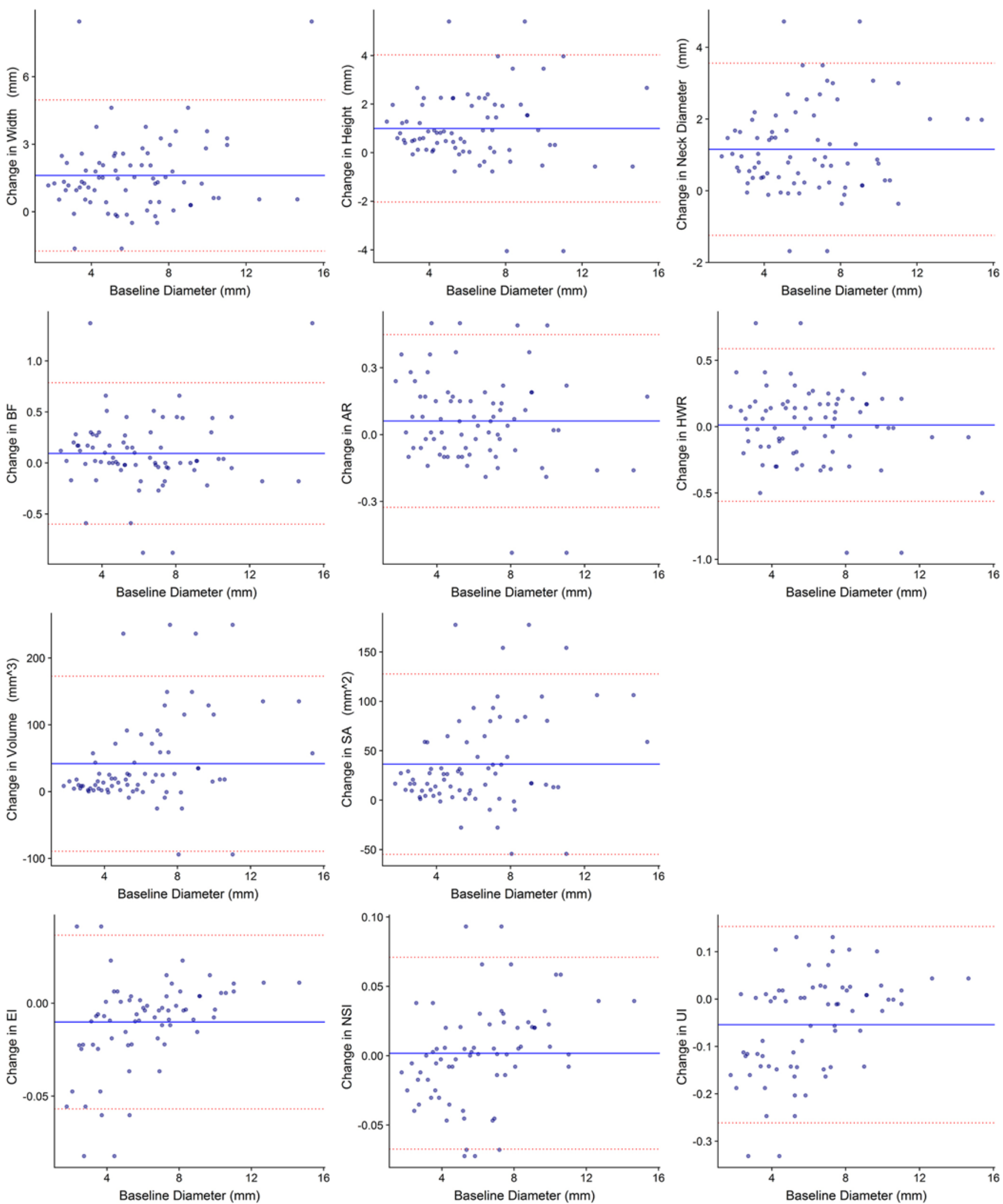

FIG. 4. Modified Bland-Altman plots showing changes in the morphological characteristics versus the baseline diameter. The blue line shows the average change, and the dotted red lines show the upper and lower border of the 95\% confidence interval. 
imaging is rarely done with 3D rotational angiography as this method is invasive.

The resolution (both in plane and in the slice direction) was around $0.5 \mathrm{~mm}$, which was our detection limit for aneurysm growth. Previous studies showed that the detection rate for aneurysm growth was high for growth $>$ $0.4 \mathrm{~mm},{ }^{26}$ but smaller enlargement might be missed. Nevertheless, these smaller enlargements are also difficult to detect on other modalities and might have limited clinical relevance.

We quantified morphological shape using the EI, UI, and NSI as defined by Raghavan et al. ${ }^{28}$ By automating the quantification method, the measurements are objective and repeatable while preserving a close connection to the current measurement methodology. Although the neckplane detection method we used, as presented by Piccinelli et al. ${ }^{27}$ has been shown to be in concordance with manually positioned planes, the algorithm sometimes misplaces the neck in wide-neck aneurysms and aneurysms with vessels originating from the aneurysms base. To prevent such errors, the measured characteristics were checked, and in case of misplacement of the neck plane, the parent vessel and aneurysm centerline were manually adjusted. Some of the measures are based on the aneurysm neck size; it has been shown that this measure differs between imaging modalities. ${ }^{30}$ In this study, we used the same modality before and after growth, thereby minimizing the differences within patients.

A selection bias is present in our study population as aneurysms with a high risk of rupture are immediately treated. This study thus only included conservative managed aneurysms. Therefore, our study population has a relative high number of small- and middle-sized middle cerebral artery aneurysms. However, the results of this study provide insight into the aneurysms that are left untreated.

\section{Conclusions}

We have shown that, during growth, the intracranial aneurysm's morphological characteristics change. This change was only significant for the size-related characteristics, the BNF, and the EI. Most size ratios and shape indices show inconsistent changes among aneurysms during growth. This suggests that, for accurate rupture prediction, morphological parameters need to be reassessed after growth. Future studies should explore the inconsistencies in morphological change among aneurysms, as different growth patterns might indicate different pathologies and therefore different rupture risks.

\section{Acknowledgments}

This study was supported by a grant from the TWIN association, Hengelo, The Netherlands.

\section{References}

1. Ajam A, Aziz AA, Asirvadam VS, Muda AS, Faye I, Safdar Gardezi SJ: A review on segmentation and modeling of cerebral vasculature for surgical planning. IEEE Access 5:15222-15240, 2017

2. Antiga L, Piccinelli M, Botti L, Ene-Iordache B, Remuzzi
A, Steinman DA: An image-based modeling framework for patient-specific computational hemodynamics. Med Biol Eng Comput 46:1097-1112, 2008

3. Backes D, Rinkel GJE, Greving JP, Velthuis BK, Murayama Y, Takao H, et al: ELAPSS score for prediction of risk of growth of unruptured intracranial aneurysms. Neurology 88:1600-1606, 2017

4. Backes D, Rinkel GJE, Laban KG, Algra A, Vergouwen MDI: Patient- and aneurysm-specific risk factors for intracranial aneurysm growth: a systematic review and meta-analysis. Stroke 47:951-957, 2016

5. Backes D, Vergouwen MDI, Tiel Groenestege AT, Bor ASE, Velthuis BK, Greving JP, et al: PHASES score for prediction of intracranial aneurysm growth. Stroke 46:1221-1226, 2015

6. Berg P, Voß S, Saalfeld S, Janiga G, Bergersen AW, ValenSendstad K, et al: Multiple Aneurysms AnaTomy CHallenge 2018 (MATCH): phase I: segmentation. Cardiovasc Eng Technol 9:565-581, 2018

7. Bijlenga P, Gondar R, Schilling S, Morel S, Hirsch S, Cuony $\mathrm{J}$, et al: PHASES score for the management of intracranial aneurysm. Stroke 48:2105-2112, 2017

8. Cebral J, Ollikainen E, Chung BJ, Mut F, Sippola V, Jahromi BR, et al: Flow conditions in the intracranial aneurysm lumen are associated with inflammation and degenerative changes of the aneurysm wall. AJNR Am J Neuroradiol 38:119-126, 2017

9. Cebral JR, Raschi M: Suggested connections between risk factors of intracranial aneurysms: a review. Ann Biomed Eng 41:1366-1383, 2013

10. de Gast AN, Soepboer A, Sluzewski M, van Rooij WJ, Beute GN: How long does it take to coil an intracranial aneurysm? Neuroradiology 50:53-56, 2008

11. Dhar S, Tremmel M, Mocco J, Kim M, Yamamoto J, Siddiqui $\mathrm{AH}$, et al: Morphology parameters for intracranial aneurysm rupture risk assessment. Neurosurgery 63:185-197, 2008

12. Etminan N, Rinkel GJ: Unruptured intracranial aneurysms: development, rupture and preventive management. Nat Rev Neurol 12:699-713, 2016

13. Fan J, Wang Y, Liu J, Jing L, Wang C, Li C, et al: Morphological-hemodynamic characteristics of intracranial bifurcation mirror aneurysms. World Neurosurg 84:114-120, 120. e1-120.e2, 2015

14. Ford MD, Hoi Y, Piccinelli M, Antiga L, Steinman DA: An objective approach to digital removal of saccular aneurysms: technique and applications. Br J Radiol 82:S55-S61, 2009

15. Gabriel RA, Kim H, Sidney S, McCulloch CE, Singh V, Johnston SC, et al: Ten-year detection rate of brain arteriovenous malformations in a large, multiethnic, defined population. Stroke 41:21-26, 2010

16. Greving JP, Wermer MJH, Brown RD Jr, Morita A, Juvela S, Yonekura M, et al: Development of the PHASES score for prediction of risk of rupture of intracranial aneurysms: a pooled analysis of six prospective cohort studies. Lancet Neurol 13:59-66, 2014

17. Guha D, Ibrahim GM, Kertzer JD, Macdonald RL: National socioeconomic indicators are associated with outcomes after aneurysmal subarachnoid hemorrhage: a hierarchical mixedeffects analysis. J Neurosurg 121:1039-1047, 2014

18. Hoffman H, Toshkezi G, Swarnkar A, Gould G, Chin LS, Krishnamurthy S: A retrospective comparison of sac and lobe morphology between ruptured and unruptured intracranial aneurysms. J Clin Neurosci 60:88-92, 2019

19. Huang ZQ, Meng ZH, Hou ZJ, Huang SQ, Chen JN, Yu H, et al: Geometric parameter analysis of ruptured and unruptured aneurysms in patients with symmetric bilateral intracranial aneurysms: a multicenter CT angiography study. AJNR Am J Neuroradiol 37:1413-1417, 2016

20. Jiang H, Shen J, Weng YX, Pan JW, Yu JB, Wan ZA, et al: Morphology parameters for mirror posterior communicating 
artery aneurysm rupture risk assessment. Neurol Med Chir (Tokyo) 55:498-504, 2015

21. Jing L, Fan J, Wang Y, Li H, Wang S, Yang X, et al: Morphologic and hemodynamic analysis in the patients with multiple intracranial aneurysms: ruptured versus unruptured. PLoS One 10:e0132494, 2015

22. Lecler A, Raymond J, Rodriguez-Régent C, Al Shareef F, Trystram D, Godon-Hardy S, et al: Intracranial aneurysms: recurrences more than 10 years after endovascular treatment-a prospective cohort study, systematic review, and meta-analysis. Radiology 277:173-180, 2015

23. Loewenstein JE, Gayle SC, Duffis EJ, Prestigiacomo CJ, Gandhi CD: The natural history and treatment options for unruptured intracranial aneurysms. Int J Vasc Med 2012:898052, 2012

24. Meng H, Tutino VM, Xiang J, Siddiqui A: High WSS or low WSS? Complex interactions of hemodynamics with intracranial aneurysm initiation, growth, and rupture: toward a unifying hypothesis. AJNR Am J Neuroradiol 35:1254-1262, 2014

25. Molyneux AJ, Birks J, Clarke A, Sneade M, Kerr RSC: The durability of endovascular coiling versus neurosurgical clipping of ruptured cerebral aneurysms: 18 year follow-up of the UK cohort of the International Subarachnoid Aneurysm Trial (ISAT). Lancet 385:691-697, 2015

26. Nakagawa D, Nagahama Y, Policeni BA, Raghavan ML, Dillard SI, Schumacher AL, et al: Accuracy of detecting enlargement of aneurysms using different MRI modalities and measurement protocols. J Neurosurg 130:559-565, 2018

27. Piccinelli M, Steinman DA, Hoi Y, Tong F, Veneziani A, Antiga L: Automatic neck plane detection and 3D geometric characterization of aneurysmal sacs. Ann Biomed Eng 40:2188-2211, 2012

28. Raghavan ML, Ma B, Harbaugh RE: Quantified aneurysm shape and rupture risk. J Neurosurg 102:355-362, 2005

29. Ruan C, Long H, Sun H, He M, Yang K, Zhang H, et al: Endovascular coiling vs. surgical clipping for unruptured intracranial aneurysm: a meta-analysis. Br J Neurosurg 29:485-492, 2015

30. Schneiders JJ, Marquering HA, Antiga L, van den Berg R, VanBavel E, Majoie CB: Intracranial aneurysm neck size overestimation with $3 \mathrm{D}$ rotational angiography: the impact on intra-aneurysmal hemodynamics simulated with computational fluid dynamics. AJNR Am J Neuroradiol 34:121128,2013

31. Schneiders JJ, Marquering HA, van den Berg R, VanBavel E, Velthuis B, Rinkel GJE, et al: Rupture-associated changes of cerebral aneurysm geometry: high-resolution 3D imaging before and after rupture. AJNR Am J Neuroradiol 35:13581362,2014

32. Schnell S, Ansari SA, Vakil P, Wasielewski M, Carr ML, Hurley MC, et al: Three-dimensional hemodynamics in intracranial aneurysms: influence of size and morphology. J Magn Reson Imaging 39:120-131, 2014

33. Taubin G: Curve and surface smoothing without shrinkage, in Proceedings of the IEEE International Conference on Computer Vision. Los Alamitos, CA: IEEE Computer Society Press, 1995, pp 852-857, 1995 (https://graphics.stanford. edu/courses/cs468-01-fall/Papers/taubin-smoothing.pdf) [Accessed May 9, 2019]
34. Thompson BG, Brown RD Jr, Amin-Hanjani S, Broderick JP, Cockroft KM, Connolly ES Jr, et al: Guidelines for the management of patients with unruptured intracranial aneurysms: a guideline for healthcare professionals from the American Heart Association/American Stroke Association. Stroke 46:2368-2400, 2015

35. Tremmel M, Dhar S, Levy EI, Mocco J, Meng H: Influence of intracranial aneurysm-to-parent vessel size ratio on hemodynamics and implication for rupture: results from a virtual experimental study. Neurosurgery 64:622-631, 2009

36. Villablanca JP, Duckwiler GR, Jahan R, Tateshima S, Martin NA, Frazee J, et al: Natural history of asymptomatic unruptured cerebral aneurysms evaluated at CT angiography: growth and rupture incidence and correlation with epidemiologic risk factors. Radiology 269:258-265, 2013

37. Xiang J, Natarajan SK, Tremmel M, Ma D, Mocco J, Hopkins LN, et al: Hemodynamic-morphologic discriminants for intracranial aneurysm rupture. Stroke 42:144-152, 2011

38. Xu J, Yu Y, Wu X, Wu Y, Jiang C, Wang S, et al: Morphological and hemodynamic analysis of mirror posterior communicating artery aneurysms. PLoS One 8:e55413, 2013

\section{Disclosures}

René van den Berg reports a consultancy with Codman DePuy Neurovascular (coil development). Henk A. Marquering reports being a cofounder of and shareholder in Nico-lab. Charles B.L.M. Majoie received a grant from the TWIN Foundation, as well as grants/grants pending from the Dutch Heart Foundation and Stryker. He is also a shareholder in Nico-lab. Money has been paid to the institution.

\section{Author Contributions}

Conception and design: Leemans, Cornelissen, Slump, Marquering, Majoie. Acquisition of data: Leemans, Cornelissen, Said, van den Berg. Analysis and interpretation of data: Leemans, Cornelissen, Said. Drafting the article: Leemans. Critically revising the article: Cornelissen, Said, van den Berg, Slump, Marquering, Majoie. Reviewed submitted version of manuscript: Cornelissen, Said, van den Berg, Slump, Marquering, Majoie. Approved the final version of the manuscript on behalf of all authors: Leemans. Statistical analysis: Leemans, Said. Administrative/technical/material support: Marquering. Study supervision: Slump, Marquering, Majoie.

\section{Supplemental Information \\ Previous Presentations}

Portions of this work were presented at the Interdisciplinary Cerebrovascular Symposium, Magdeburg, Germany, June 6-8, 2018.

\section{Correspondence}

Eva L. Leemans: Amsterdam UMC, Amsterdam, The Netherlands.e.1.leemans@amc.uva.nl. 